\title{
hTERT promotes the invasion of telomerase-negative tumor cells in vitro
}

\author{
SONG-TAO YU*, LING CHEN* ${ }^{*}$, HONG-JIN WANG, \\ XU-DONG TANG, DIAN-CHUN FANG and SHI-MING YANG \\ Institute of Gastroenterology, Southwest Hospital, \\ Third Military Medical University, Chongqing 400038, P.R. China
}

Received February 23, 2009; Accepted May 11, 2009

DOI: 10.3892/ijo_00000344

\begin{abstract}
Telomerase activation is a common feature of most types of human cancers. Although several studies have shown that activation of telomerase might participate in the progression of tumors, the mechanism by which telomerase activation causes the invasion and metastasis of tumors remains unclear. In this study, we transfected a vector containing the full-length cDNA of hTERT into a telomerasenegative osteosarcoma cell line U2OS (hTERT/U2OS). Vacant vector-transfected U2OS cells served as a control (EGFP/U2OS). We then compared the biological and vitodynamic changes in these transfected and untransfected U2OS cells. The hTERT protein was detected in hTERT/U2OS cells by Western blot analysis and immunochemistry assay. The telomere length in hTERT/U2OS cells was longer than that in EGFP/U2OS and untransfected U2OS cells. We also found using vacuum micropipette aspiration that hTERT transfection did not only promote the proliferation of hTERT-transfected U2OS cells but also increased the cellular adhesion capacity to the extracellular matrix. Transwell matrigel assay confirmed an increased invasion ability in hTERT/U2OS cells. These results strongly suggest that hTERT transfection promotes the invasion of telomerase-negative cells. Telomerase-
\end{abstract}

Correspondence to: Dr Shi-Ming Yang, Institute of Gastroenterology of PLA, Third Military Medical University, Chongqing 400038, P.R. China

E-mail: shimingyang@yahoo.com

*Contributed equally

Abbreviations: hTERT, human telomerase reverse transcriptase; ALT, alternative lengthening of telomeres; RTL, relative telomere length

Key words: human telomerase reverse transcriptase, alternative lengthening of telomeres, invasion, relative telomere length, U2OS, metastasis mediated telomere maintenance enables these cells to achieve a fully malignant endpoint, including invasion and metastasis.

\section{Introduction}

A telomere is an overhang DNA sequence at chromosomal ends containing tandem repeats of 5'-GGTTAG-3', which forms a three-dimensional cap preventing recognition as DNA breaks $(1,2)$. Since telomeres continuously shorten in most somatic cells during their life span, a telomerase complex helps to lengthen it in cells with high replicative demands, such as germ cells and stem cells (3). Telomerase is a unique ribonucleoprotein that mediates the RNA-dependent synthesis of telomeric DNAs. The human telomerase complex contains more than 30 proteins, most of which dissociate during the activation process (4). Telomerase is found to be repressed in most somatic cells, but it is highly expressed in $90 \%$ of tumors (5). Although telomerase does not cause cancer per se, up-regulation of telomerase is necessary for maintaining the immortality of tumor cells (6). Human telomerase reverse transcriptase (hTERT), an important subunit of telomerase, is the rate-limiting component of the telomerase complex, and it is most closely correlated with telomerase activity. Recent data have shown that telomerase and its hTERT subunit are implicated in the entire process of tumorigenesis. Our previous study showed that hTERT was expressed, not only in gastric cancer, but also in premalignant lesions such as chronic atrophy gastritis, intestinal metaplasia and dysplasia (7). Moreover, we found that expression of hTERT was correlated with the invasion depth of tumors $(8,9)$. These results indicate that hTERT participates in tumorigenesis from the very beginning of transformation to invasion and metastasis.

It was widely believed for many years that telomerase was the only telomere lengthening mechanism involved in cell aging and tumorigenesis, until the unveiling of the alternative lengthening of telomere (ALT) mechanism $(10,11)$, which is characterized by instability at a specific minisatellite locus and high rates of telomeric recombination exchange $(12,13)$. Telomere maintenance in approximately $10 \%$ of tumors relies on the ALT mechanism. Studies have indicated that, while telomerase was found to be a telomere maintenance mechanism in most epithelial tumors, the ALT mechanism 
exists in most tumors with mesenchymal origin such as osteosarcoma tumors (14) and glioblastoma multiforme (10).

Although telomerase and ALT are involved in the maintenance of telomeres, malignant phenotyes are significantly different in these two types of cells. Chang and colleagues (15) confirmed that, although telomere dysfunction provokes chromosomal aberrations that initiate carcinogenesis, telomerase-mediated telomere maintenance enables such initiated cells to efficiently achieve a fully malignant endpoint, including metastasis. Another model showed that constitutive expression of TERT in thymocytes and peripheral $\mathrm{T}$ cells promotes lymphoma. Notably, such lymphomas were more disseminated than those in wild-type controls, and they affected both lymphoid and non-lymphoid tissues without any correlation to telomere length (16). Stewart and colleagues (17) found that the transfection of TERT enabled an ALT cell line to form a subcutaneous tumor, which could not be achieved before transfection. In another study, telomerase-negative human and bovine cells were found to have progressively slower growth and decreased invasion and metastasis when serially transplanted in immunodeficient mice, but re-acquired tumorigenicity by expressing TERT (18).

Thus, it appears evident from the above studies that telomerase takes part in the entire process of tumorigenesis, and introducing telomerase into an ALT cell line promotes or enhances tumor invasion and metastasis. However, the cellular mechanism of this outcome remains unclear. In this study, in order to explore the role of hTERT in the invasion and metastasis of tumors at the cellular level, we transfected a vector containing the full-length cDNA of hTERT into an ALT osteosarcoma cell line U2OS and compared a series of biological and vitodynamic changes in the transfected and untransfected U2OS cells. We found that hTERT transfection increased the proliferative and invasive ability of U2OS cells. Using a micropipette aspiration test, we found that hTERTtransfected cells showed enhanced adhesion ability to the extracellular matrix, which may explain the initiation step of basement membrane invasion and remote colonization. A transwell invasion test confirmed the enhanced invasion ability in hTERT-transfected cells. These results strongly suggest that hTERT gene expression in an ALT cell line increases proliferation ability and enhances adhesion capacity to the extracellular matrix, which may lead to an increased invasion capacity of tumor cells.

\section{Materials and methods}

Cell line. A telomerase-negative cell line (U2OS human osteosarcoma) was obtained from the American Type Culture Collection (ATCC) and was maintained in a logarithmic growth phase at $37^{\circ} \mathrm{C}$ in a $5 \% \mathrm{CO}_{2}$ humidified atmosphere in its own culture medium, McCoy's 5a (ATCC) supplemented with $10 \%$ fetal calf serum (FCS).

Vector construction and transfection. Plasmid pGRN145 (a kind gift from Dr Kevin Kaster, Geron Inc., USA) contained 3.45-kb hTERT cDNA between the two EcoRI restriction endo-nuclease sites and was 14-kb long. pIRES2-EGFP was purchased from BD Biosciences (Clontech, USA). The fragment of hTERT was digested from pGRN145 with EcoRI and ligated into the site of EcoRI of pIRES2-EGFP. The orientation of recombinant DNA was further confirmed by NotI restriction endonuclease. The sense vector, pIRES2EGFP-hTERT, was obtained and transfected into U2OS cells using Lipofectamine 2000 (Invitrogen, CA, USA). The other group was transfected simultaneously with pIRES2-EGFP as a blank control. Seventy-two hours after transfection, antibiotic G418 (Amresco, OH, USA) was used at a 2-day descending concentration from 700 to $200 \mu \mathrm{g} / \mathrm{ml}$ medium. A stable expression cell clone formed 2 weeks after being selected by G418 and was removed to a new culture dish for amplification. The groups of transfected cells were named hTERT/ $\mathrm{U} 2 \mathrm{OS}$ and EGFP/U2OS, respectively.

hTERT protein detection. Expression of the hTERT protein was detected by immunohistochemistry and Western blot analysis as described in a previous study (19). For the immunohistochemistry, cells from three groups (U2OS, hTERT/ U2OS and EGFP/U2OS) were grown on a slide coated with polylysine and fixed for $10 \mathrm{~min}$ in $4 \%$ para-formaldehyde (Sigma, St. Louis, MO, USA). The slides were then washed with buffer containing $0.1 \mathrm{M}$ Tris ( $\mathrm{pH} 7.5$ ), $1.5 \mathrm{M} \mathrm{NaCl}$ and $1 \%$ BSA and permeabilized in the presence of $2 \%$ Triton X-100. Next, cells were incubated with hTERT antibody $(0.5 \mu \mathrm{g} / \mathrm{ml}$; Santa Cruz Biotechnology, CA, USA) for $1 \mathrm{~h}$ at room temperature (RT), washed and further incubated with secondary antibody. Finally, the cells were incubated for $15 \mathrm{~min}$ with an avidin-biotin enzyme reagent. The slides were then immersed in $\mathrm{DAB} / \mathrm{H}_{2} \mathrm{O}_{2}$ solution for stain development. PBS was used as a negative control in place of the primary antibody.

For Western blot analysis, proteins in the cell extracts of each group were separated by sodium dodecyl sulfatepolyacrylamide gel electrophoresis (SDS-PAGE) through an $8 \%$ polyacrylamide gel and then transferred onto a nitrocellulose membrane. The membrane was incubated with $5 \%$ non-fat milk in PBS and then with anti-hTERT mAb for $2 \mathrm{~h}$ at RT. The membranes were washed and incubated with an alkaline phosphatase-conjugated goat anti-mouse IgG antibody (Amersham Biosciences, Buckinghamshire, UK) for $1 \mathrm{~h}$ at room temperature. Immunoreactive bands were detected using the ECL Western Blot Analysis System (Amersham Biosciences).

TRAP assay for telomerase activity. Telomerase activity of the three U2OS groups was detected with a TRAP assay kit (DingGuo, Beijing, China). The procedure was performed according to the manufacturer's instructions. Gastric adenocarcinoma cell line KATO III was included as a positive control. Briefly, $5 \times 10^{6} \mathrm{U} 2 \mathrm{OS}$ cells from each group were collected and lysed on ice with $1 \mathrm{ml}$ of buffer A for $15 \mathrm{~min}$. The suspension was then centrifuged at 12,000 rpm for $5 \mathrm{~min}$, and the supernatant was discarded. Buffer B (100 or $200 \mu \mathrm{l}$ ) was added to the tube and thoroughly mixed. After $30 \mathrm{~min}$, the mixture was centrifuged at $12,000 \mathrm{rpm}$ for $15 \mathrm{~min}$, and the supernatant was collected. The supernatant $(2 \mu 1)$ was added into $20 \mu \mathrm{l}$ of the PCR mixture for a 30-min incubation at $20^{\circ} \mathrm{C}$ and then a $3-\mathrm{min}$ incubation at $90^{\circ} \mathrm{C}$. One microliter of CX primer and $1 \mu \mathrm{l}$ of Taq polymerase and two drops of 
paraffin oil were added to the PCR tube followed by 35 cycles of the following profile: $90^{\circ} \mathrm{C}$ for $45 \mathrm{sec}, 50^{\circ} \mathrm{C}$ for $45 \mathrm{sec}$ and $72^{\circ} \mathrm{C}$ for $60 \mathrm{sec}$. Fifteen microliters of the PCR product was loaded and run on $12.5 \%$ polyacrylamide gel. After electrophoresis, the gel was stained with SYBR Green II (Sigma Aldrich, USA).

Fluorescence quantitative PCR for relative telomere length. Relative telomere length (RTL) of the three groups of U2OS cells were measured with a quantitative PCR-based technique first described by Cawthon (20). For each sample, the cycle threshold $(\mathrm{Ct})$ value of telomere PCR was used in comparison with the Ct of 36b4 PCR, a single copy gene that was used as a reference, and the ratio was defined as the $\mathrm{T} / \mathrm{S}$ ratio. The final relative $\mathrm{T} / \mathrm{S}$ ratio of each sample, which corresponded to the relative telomere length of its DNA, was calculated by comparing the T/S ratio of each sample DNA to the T/S ratio of a reference DNA and a DNA sample pooled from the three groups of U2OS cells (21). The PCR strategy was as follows. Duplicate DNA samples were amplified in parallel 50- $\mu 1$ PCR reactions. For telomere amplification, 270 and $900 \mathrm{nM}$ of forward and reverse telomere primer was used in each 50- $\mu$ l reaction volume: (forward, 5'GGTTTTTGAGGGTGA GGGTGAGGGTGAGGGTGAGGGT3', reverse, 5'TCCC GACTATCCCTATCC CTATCCCTATCCCTATCCCTA3'), and for 36b4 amplification, 300 and $500 \mathrm{nM}$ of forward and reverse $36 \mathrm{~b} 4$ primer was used: (forward, 5'CAGCAAGTGG GAAGGTGTAATC C3', reverse, 5'CCCATTCTATCATCA ACGGGTACAA3'). All PCRs were conducted in a Corbett Research Rotor-Gene 3000 Real-time Thermal Cycler (Corbett Research, Cambridge, UK). The thermal cycling profile for both amplicons began with a $95^{\circ} \mathrm{C}$ incubation for 10 min to activate the Platinum Taq DNA polymerase. For the telomere PCR, this was followed by 35 cycles of $15 \mathrm{sec}$ at $95^{\circ} \mathrm{C}$ and $2 \mathrm{~min}$ at $54^{\circ} \mathrm{C}$. For the $36 \mathrm{~b} 4 \mathrm{PCR}, 35$ cycles of $15 \mathrm{sec}$ at $95^{\circ} \mathrm{C}$ and $1 \mathrm{~min}$ at $58^{\circ} \mathrm{C}$ was performed. For optimization of the assays, a dilution series (200-0.02 ng DNA, 10-fold dilution, 5 points) was run for both the telomeres and 36b4 PCRs, in which a linearity $\left(\mathrm{R}^{2}>0.99\right)$ over this range of input DNA was noted. Since the relative T/S ratio will reflect RTL only when 36b4 is equally amplified from each sample and reference group, we determined the relative ratio of $36 \mathrm{~b} 4$ gene copies to $\beta$-globin gene copies by Q-PCR using the following primers as described by Cawthon: $\beta-$ globin forward, 3'-GCTTCTGACACAACTGTGTTCAC TAGC-5' and reverse, 3'-CACCAACTTCATCCACGTT CACC-5' (400 nM each). The specificity of all amplifications was determined by melting curve analysis.

Cell cycle analysis by flow cytometry. Distribution of the cell cycle was detected by flow cytometry as previously reported (21). Cells at $50 \%$ confluency or less were fixed by the addition of $70 \%$ ice-cold ethanol for at least $24 \mathrm{~h}$. The cells were washed with PBS and treated with RNase A (75 U/ml) for $30 \mathrm{~min}$ at $37^{\circ} \mathrm{C}$, washed again in PBS, and resuspended in PBS containing $15 \mathrm{mg} / \mathrm{ml}$ propidium iodide. A minimum of $2 \times 10^{4}$ cells was analyzed with a Coulter Epics Profile II (Coulter Electronics, Inc., Hialeah, FL, USA). The proliferative index (PI) was calculated according to the following formula: $\left[\left(S+G_{2} M\right) /\left(G_{0} / G_{1}+S+G_{2} M\right)\right] \times 100 \%$.
Cell proliferation by MTT. A modified 3-(4,5-dimethylthiazol-2-yl)-2,5-diphenyltetrazolium-bromide (MTT) technique was adopted to determine the cell proliferation and viability of each group as previously reported (22).

Cell adhesion assay by vacuum micropipette aspiration. A vacuum micropipette aspiration technique was applied to test the adhesion strength of U2OS cells on a basement membrane substitute as previously reported $(23,24)$. U2OS cells from each group were seeded on a transparent chamber with a $2-\mathrm{cm}^{2}$ flat bottom. Before seeding the cells, the bottom surface of the chamber was coated with a soluble basement membrane extract, Cultrex BME (R\&D Systems, Minneapolis, MN, USA). Five hundred microliters of $9 \mathrm{mg} / \mathrm{ml}$ Cultrex BME was added followed by $1-4 \mathrm{~h}$ of cell incubation at $37^{\circ} \mathrm{C}$ in a $5 \% \mathrm{CO}_{2}$ atmosphere. The aspiration test was performed under a microscope-monitored micromanipulator that approaches the micropipette to the cell surface. A negative pressure controller provides 5-10 $\mathrm{mmHg}$ of stepwise negative pressure for pulling the cell. The critical pressure at which the cell detaches from the chamber bottom indicates its adhesion strength according to the equation $\mathrm{T}=\Delta \mathrm{P} \times \pi \times(\mathrm{Rp} / \mathrm{Rc})^{2} \mathrm{x}$ $\cos \theta$ (Fig. 5A), where Rp and Rc refer to the radius of the micropipette and the adhering area between the cell and chamber bottom, respectively, and $\theta$ refers to the included angle between the micropipette and the bottom surface. In this study, 25 random cells of each group were measured, and the adhesion strength was obtained for each group of U2OS cells.

Cell invasion by transwell matrigel assay. The invasion potential of the three U2OS groups was measured by a transwell method as previously reported (21). Before the invasion assay, the NIH-3T3 cell culture supernatant was prepared for use as a chemoattractant. Three groups of U2OS cells were seeded into a polycarbonate $8-\mu \mathrm{m}$ pore Costar Transwell filter (Corning, NY, USA) which was coated with $50 \mu \mathrm{l}$ of $1 \mathrm{mg} / \mathrm{ml}$ Matrigel (BD Biosciences, NJ, USA) before the seeding and prehydrated with culture solution at $37^{\circ} \mathrm{C}$ in an incubator for $2 \mathrm{~h}$. For each U2OS group, duplicates of $3 \times 10^{5}$ cells per insert were seeded into the upper chamber of the transwell with culture solution that contained no serum. The transwell was placed in a 24 -well plate that contained $300 \mu \mathrm{l}$ of NIH-3T3 supernatant and $300 \mu \mathrm{l}$ of McCoy's 5a culture solution with $10 \%$ fetal calf serum. After $24 \mathrm{~h}$ of invasion in a humidified atmosphere of $95 \%$ air and $5 \% \mathrm{CO}_{2}$ at $37^{\circ} \mathrm{C}$, the upper surface of the filter was gently washed with PBS and erased with a cotton pledget. Ethanol (95\%) was added to the lower chamber for 5-10 min of cell dehydration, and subsequently $2 \mathrm{ml}$ of $1 \%$ tetrabromofluorescein dye was added to the lower chamber for 10-20 min. The polycarbonate membrane was then cut, and the invasive cells on the lower surface of the filter were counted in five random fields.

Statistics. All experiments were run in triplicate, and the results are presented as the mean \pm SD of triplicate determinations. Statistical analysis was performed using the Student's t-test. Differences were considered statistically significant at a P-value $<0.05$. All statistical analyses were carried out with SPSS 11.5 software. 


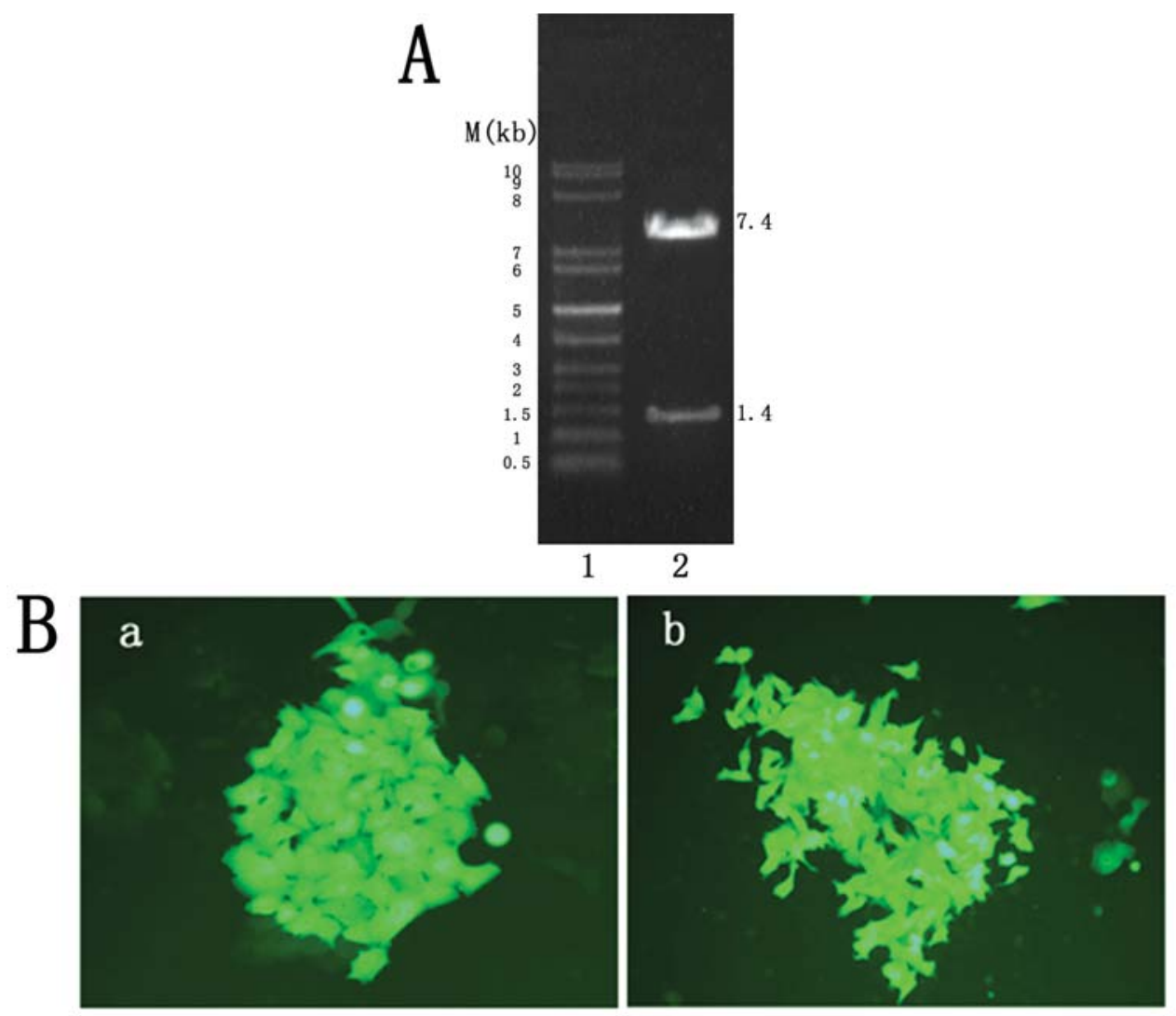

Figure 1. Vector construction and stable cell clone screening. The vector was digested with NotI restriction endonuclease. (A) Sense hTERT expression vector formed 2 fragments $(7.4$ and $1.4 \mathrm{~kb}$ ) after electrophoresis, which was in accordance with the designed construction. After selection by G418 for 2 weeks, drugresistant clones were isolated and named hTERT/U2OS and EGFP/U2OS, respectively. Since the pIRES2-EGFP plasmid encodes enhanced green fluorescent protein (EGFP), when the pIRES2-EGFP-hTERT- or pIRES2-EGFP-expressing vectors are successfully transduced into the U2OS cells, they were detected by fluorescence microscopy. (B) The EGFP protein was detected in hTERT/U2OS (a) and EGFP/U2OS (b).

\section{Results}

Vector construction and transfection. The 3.45-kb hTERT fragment was digested from pGRN145 by EcoRI and inserted into pIRES2-EGFP. The plasmid was confirmed by digestion with NotI. A 1.4- and 7.4-kb band represented a sense recombinant (Fig. 1A). Further sequencing was applied to verify the orientation of the recombinant vectors (data not shown). The sense plasmid was named pIRES2-EGFPhTERT and was transfected into U2OS. The plasmid of pIRES2-EGFP was transfected simultaneously as a blank control. A stable cell clone with high expression of EGFP formed after 2 weeks of selection by G418 (Fig. 1B). The following experiments were carried out in the hTERT/U2OS, EGFP/U2OS and U2OS parental cells.

hTERT protein detection. In order to detect the expression of hTERT protein in transfected and untransfected U2OS cells, immunohistochemistry and Western blot analysis were used. We found that hTERT/U2OS cells had a significant brown signal in the cytoplasm and nucleus, while EGFP/U2OS and untransfected U2OS had no positive signal in the entire slide upon immunohistochemistry (Fig. 2A). Western blot analysis indicated that hTERT/U2OS cells had a band of $120 \mathrm{kDa}$ for hTERT expression, while EGFP/U2OS and untransfected U2OS cells displayed no positive signal. The $36 \mathrm{kDa}$ of GAPDH is present in each group (Fig. 2B).
TRAP assay for telomerase activity. We used the TRAP assay to detect the telomerase activity in transfected and untransfected U2OS cells. Telomerase activity was detected in hTERT/U2OS, which was similar to the positive control cells KATO III. EGFP/U2OS and untransfected U2OS did not show telomerase activity (Fig. 3A). This result verified that transfection of hTERT into an ALT cell line resulted in activated telomerase expression.

Quantitative PCR for telomere length. Relative telomere length (RTL) was measured by quantitative PCR. The result showed that the relative telomere length of hTERT/U2OS, EGFP/U2OS and untransfected U2OS was 3.133, 1.720 and 1.643, respectively (Fig. 3B). For both telomere and 36b4 amplification, a serially diluted sample was run, and a linearity of $\mathrm{R}^{2}>0.99$ was noted. The specificity of all amplification was determined by melting curves (data not shown). The relative ratio of $36 \mathrm{~b} 4 / 3$-globin copy number indicated that equal copy numbers of the $36 \mathrm{~b} 4$ gene per cell were amplified in all DNA samples (data not shown).

Flow cytometry of the cell cycle. A decreased percentage of cells in the $\mathrm{G}_{0} / \mathrm{G}_{1}$ phase and an increased percentage of cells in the proliferative index (PI) were found in hTERT-transfected U2OS cells when compared with the parental and vacant vector-transfected U2OS cells (Table I). This indicated that hTERT transfection promotes the proliferation of U2OS cells. 


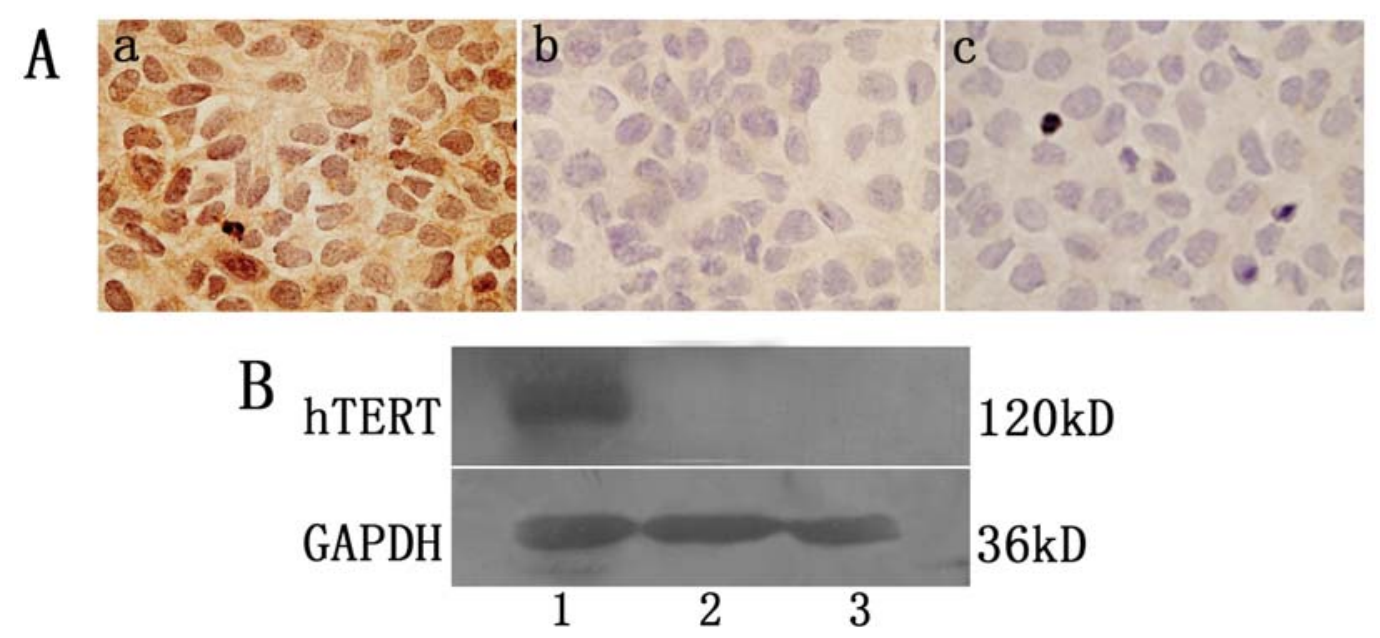

Figure 2. Expression of hTERT protein in transfected and untransfected U2OS cells. (A) Immunohistochemical staining for hTERT expression in transfected and untransfected U2OS cells (x400). hTERT protein was expressed in hTERT/U2OS (a), while EGFP/U2OS (b) and untransfected U2OS (c) showed no detectable expression. (B) Western blot analysis for hTERT expression in transfected and untransfected U2OS cells. The hTERT protein was expressed in hTERT/U2OS cells, but not in EGFP/U2OS and untransfected U2OS cells. Lane 1, hTERT/U2OS cells; lane 2, EGFP/U2OS cells; lane 3, U2OS cells.
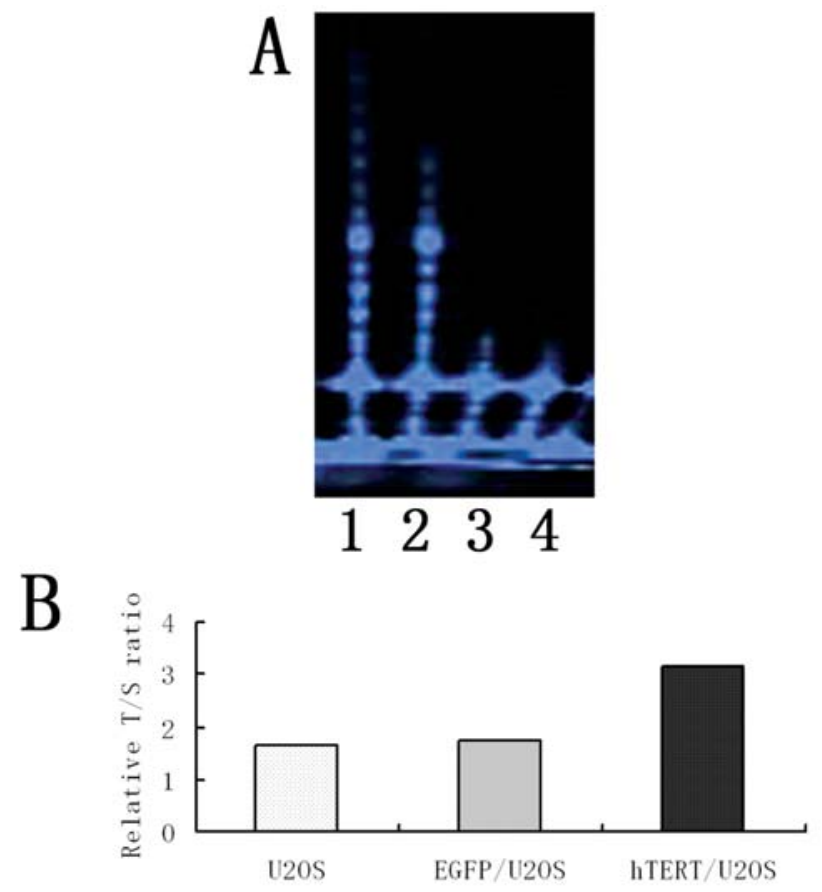

Figure 3. Effect of hTERT transfection on telomerase activity and relative telomere length in U2OS cells. (A) Telomerase activity of transfected and untransfected U2OS cells as detected by the TRAP assay. Lane 1, KATO III gastric cancer cells; lane 2, hTERT/U2OS cells; lane 3, EGFP/U2OS cells; lane 4 , U2OS cells. (B) Relative telomere length detected by quantitative PCR. For optimization of the quantitative PCR, a dilution series $(0.2-200 \mathrm{ng}$ DNA, 10-fold dilution, 5 points) was run for both $36 \mathrm{~b} 4$ and telomere PCR, in which a linearity $\left(\mathrm{R}^{2}>0.99\right)$ over this range of input DNA was noted. The relative telomere length was calculated by the equation $\mathrm{RTL}=$ relative $\mathrm{T} / \mathrm{S}$ ratio $=$ sample $[\mathrm{Ct}($ telomere $) / \mathrm{Ct}(36 \mathrm{~b} 4)] /$ reference $[\mathrm{Ct}($ telomere $) / \mathrm{Ct}(36 \mathrm{~b} 4)]$.

MTT measurement for cell proliferation. The average absorbance rate of 6 wells of each group was measured for 7 days and marked as 7-point cell growth curves. The three
Table I. Alteration of cell cycle distribution in transfected and untransfected U2OS cells.

\begin{tabular}{lcccc}
\hline Cell lines & $\mathrm{G}_{0} / \mathrm{G}_{1}(\%)$ & $\mathrm{G}_{2} / \mathrm{M}(\%)$ & $\mathrm{S}(\%)$ & $\mathrm{PI}(\%)$ \\
\hline U2OS & 57.7 & 22.3 & 20.0 & 42.3 \\
EGFP/U2OS & 59.9 & 21.6 & 18.6 & 40.2 \\
hTERT/U2OS & 50.4 & 10.6 & 39.0 & 49.6 \\
\hline
\end{tabular}

curves showed that the hTERT/U2OS group had a higher average absorbance rate than the EGFP/U2OS and untransfected U2OS group at each point, with a progressive difference from day 1 to 7 , which proved that the proliferation of hTERT-modified ALT tumor cells was enhanced compared with the blank and unmodified controls (Fig. 4).

Micropipette aspiration for cell adhesion. The cell adhesion of 25 random cells of each group were measured, and the average adhesion was calculated. An average adhesion of $(967.6 \pm 102.6) \times 10^{-10} \mathrm{~N}$ was recorded for the hTERT/U2OS group, while, notably, the EGFP/U2OS and untransfected U2OS groups had only $(694.8 \pm 105.6) \times 10^{-10}$ and $(707.8 \pm 74.2)$ $\mathrm{x} 10^{-10} \mathrm{~N}$ of adhesion, respectively (Fig. 5B). The introduction of hTERT into an ALT cell line enhanced its adhesion strength with the extracellular matrix. This alteration probably plays a role in the initiation of tumor invasion. The effect of the alteration of adhesion was investigated in the invasion assay.

Cell invasion assay. After a 24-h invasion, cells on the lower surface of the filter were fixed and dyed. Five random fields of each group were counted for invaded cells. An average of $154.8 \pm 5.2$ cells was recorded in the hTERT/U2OS group, 


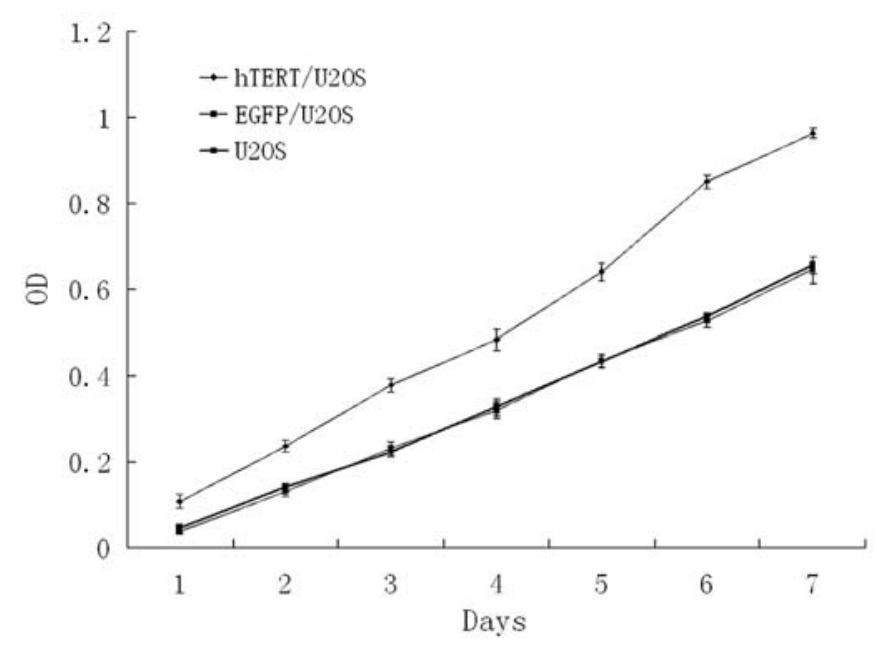

Figure 4. Effect of hTERT transfection on cell proliferation in transfected and untransfected $\mathrm{U} 2 \mathrm{OS}$ cells.

which was significantly higher than that in the EGFP/U2OS $(56.2 \pm 7.5, \mathrm{p}<0.05)$ and untransfected U2OS $(58.4 \pm 4.2$, $\mathrm{p}<0.05)$ groups (Fig. 6).

\section{Discussion}

Infinite proliferation and invasion are the two most critical properties of malignant tumors. Telomerase is responsible for elongating telomeres in tumor cells. Increasing evidence over the past ten years suggests that telomerase plays an important role in tumorigenesis and cancer development. As the most important subunit, hTERT is the rate-limiting component of the telomerase complex and is most closely correlated with telomerase activity. When hTERT was suppressed in cells, short-term telomere integrity was not affected, but the overall configuration of chromatin was disturbed. Moreover, telomerase was found to induce cell proliferation by affecting the expression of growth-promoting genes such as epidermal growth factor receptor (EGFR) (25). Geserick et al demonstrated that mouse telomerase reverse transcriptase (mTERT) reduces the proliferative potential of primary mouse embryonic fibroblasts by repressing growth-inhibiting genes of the transforming growth factor- $\beta$ (TGF- $\beta$ ) signalling network (26). In the present study, we also found that hTERT-transfected U2OS cells exhibited a stronger proliferation ability. Unlike most tumor cells that express hTERT, U2OS cells obtain infinite proliferation ability without the expression of hTERT. The alteration of the cell cycle and the consequent increase in proliferation ability in hTERT-transfected U2OS cells was therefore attributed to the re-activation of hTERT.

Recent data have also indicated an increasing connection between telomerase and tumor invasion, as well as metastasis (27). In a previous study, a telomerase-negative tumor model was constructed and confirmed as a fully malignant phenotype. However, when serially transplanted, tumors showed progressively slower growth, and decreased invasion and metastasis. The expression of hTERT in these tumors enabled

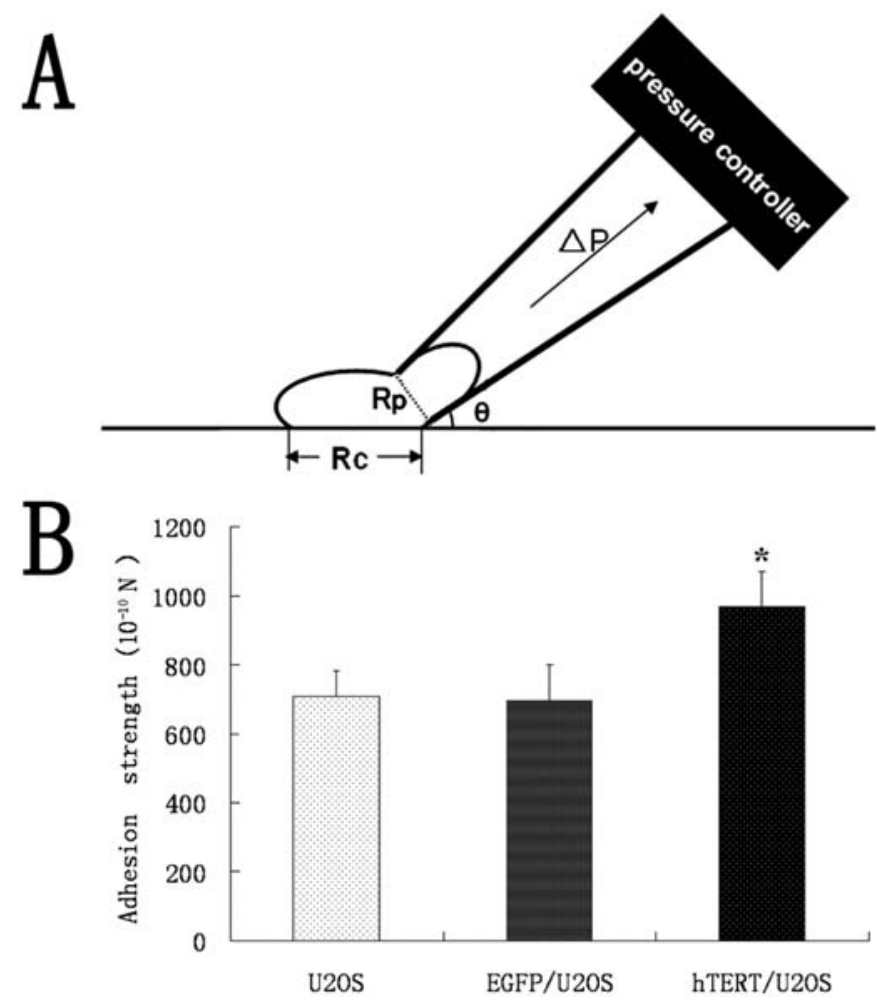

Figure 5. Alteration of cell adhesion in hTERT-transfected U2OS cells by micropipette aspiration. (A) Model of micropipette aspiration. A pressure controller provided $10 \mathrm{mmHg}$ of stepwise negative pressure that aspirates cells at a certain angle $\theta$. The critical pressure, $\Delta \mathrm{P}$, was defined as the pressure at which point the cell detaches from the surface. Thus, the horizontal distribution of the micropipette's pulling strength indicates the cell adhesion strength to the surface, which was calculated according to the equation $\mathrm{T}=\mathrm{P} \times \pi \times(\mathrm{Rp})^{2} \times \cos \theta$, where $\mathrm{Rp}$ is the caliber of the micropipette. The adhesion strength was compared to the contact surface of the cell with the well for the final relative adhesion strength using $\mathrm{T}=\Delta \mathrm{P} \times \pi \times(\mathrm{Rp} / \mathrm{Ra})^{2} \times \cos \theta$. For each sample, 25 random cells were measured and the mean adhesion strength was calculated. (B) The average adhesion strength in transfected and untransfected U2OS cells was statistically significant at $\mathrm{p}<0.05$ using a paired Student's t-test when compared with the U2OS and EGFP/U2OS groups.

them to re-acquire tumorigenicity (18). Bagheri et al showed that telomerase activity controls the glycolytic pathway in melanoma, potentially altering the energy state of tumor cells, thereby promoting the invasion and metastasis of tumors (28). Indeed, telomerase plays a certain role in tumor invasion and metastasis, but the cellular basis of this involvement has rarely been investigated. Here, we used ALT cell line U2OS as a telomerase-negative control and transfected it with a vector that contained the full-length cDNA of hTERT. A screened stable cell clone then underwent a cytomechanics test in order to determine the cell adhesion ability with the basement membrane which constitutes the main barrier of basal invasion and remote colonization. A significant increase in the adhesion ability of hTERT-transfected cells was noted. To further elucidate the consequence of this alteration by vitodynamics, cells underwent a transwell invasion test, which showed a much higher invasion rate in hTERT-transfected cells than in controls. This result strongly suggests that the introduction of hTERT alters cell interaction 


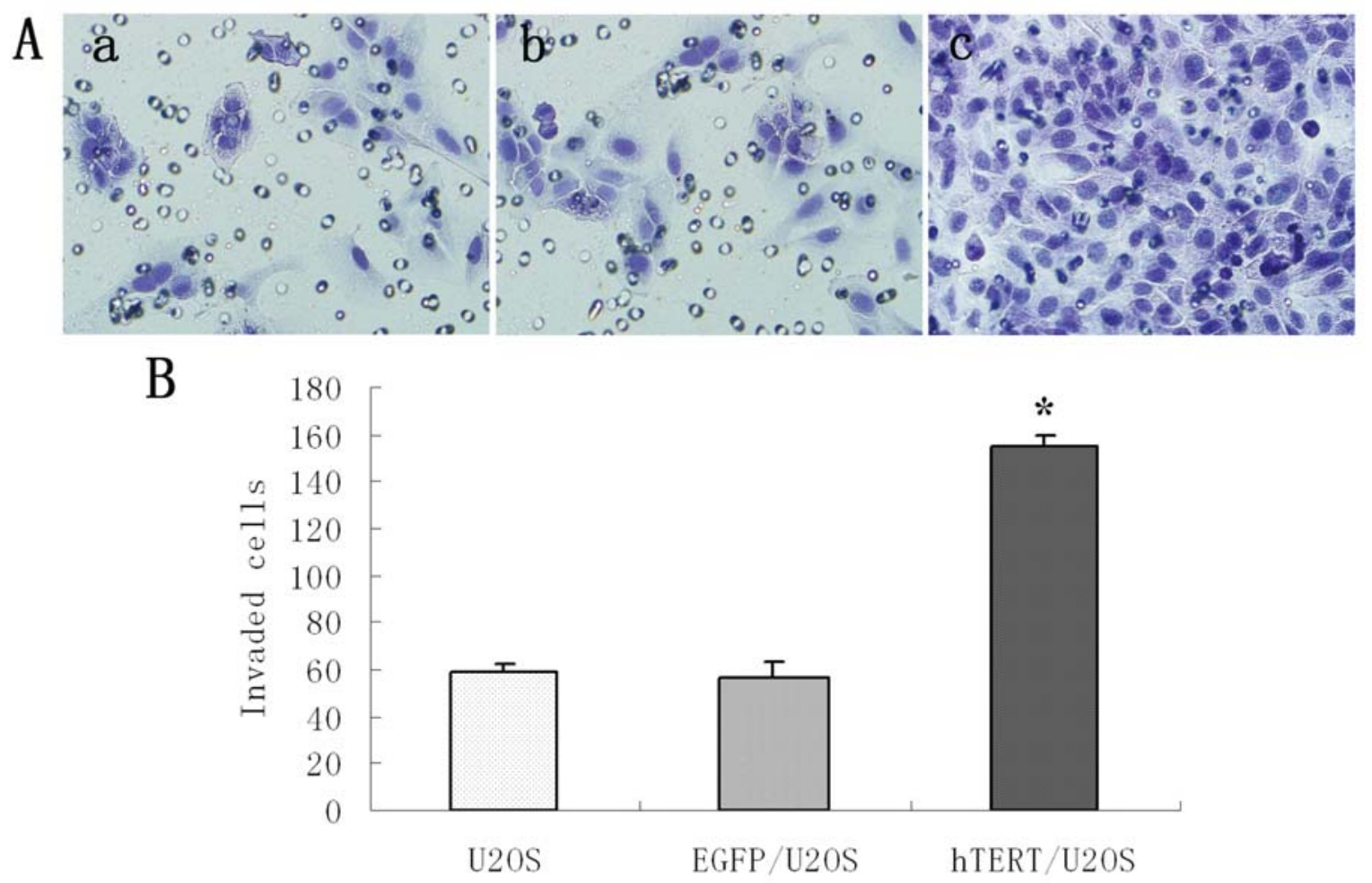

Figure 6. Effect of hTERT transfection on cell invasion in U2OS cells. (A) Invasion of transfected and untransfected U2OS cells (x200): a, U2OS; b, EGFP/ U2OS; c, hTERT/U2OS. (B) Alteration of invasion capacity in transfected and untransfected U2OS cells. Data were statistically significant ( $<<0.05$; paired Student's t-test) when compared with the U2OS and EGFP/U2OS groups.

with the extracellular matrix, which results in enhanced adhesion leading to substantial invasion and metastasis.

Based on the above analysis, the question was raised as to whether the contribution of telomerase to tumor invasion and metastasis can be attributed to its ability to elongate telomeres. Tumor cells utilizing ALT contain long heterogeneous telomeres ranging from $<3$ to $>50 \mathrm{~kb}$ in length. Chang et al found that ALT tumor cells failed to give rise to macroscopic lung metastasis despite their acquired robust growth in culture and the vigorous ability to generate subcutaneous tumors. After hTERT transfection, these ALT tumor cells began to form macroscopic lung metastases. These results strongly indicate that ALT-mediated telomere maintenance is not equivalent to telomerase activity in the progression of tumors, especially invasion and metastasis (15). In the present study, telomere length in U2OS cells was extended after transfection with the hTERT gene, and the transfected cells exhibited more proliferation and invasion ability. Thus, we presumed that re-activation of telomerase activity, rather than telomere elongation, restricted these malignant phenotypes. Although ALT tumor cells transfected with hTERT demonstrate more invasive and metastatic potential, the mechanisms remain unclear. In the present study, we found that hTERT transfection increased adhesion strength to the extracellular matrix. Increased adhesion creates a vital condition for tumor cells invading through the basement membrane and colonizing at remote sites (29). This altered adhesion may be a critical contributor that boosts U2OS cell invasion and metastasis. These findings provide a novel insight into cancer metastasis and may have potential implications in anticancer drug discovery and development. Thus, from the point of view of vitodynamics, we propose that the enhanced invasive ability of hTERT/U2OS cells is attributable to the increased adhesion ability of these cells.

In summary, we demonstrated at the cellular level that hTERT transfection in an ALT cell line re-activated its telomerase activity and further promoted its invasive and metastatic potential. The mechanism to enhance these malignant phenotypes was correlated with the increasing adhesion ability of these cells to the extracellular matrix after hTERT transfection. Our data provide new insight into the recognition of telomerase and ALT in tumor cells and also suggest some possible differences between ALT-mediated and telomerase-mediated tumor cells. In view of the current interest in developing anti-cancer therapeutics directed against telomerase, a constant concern is whether resistance will emerge due to the activation of ALT after telomerase activity is inhibited. The present study demonstrated that ALT-related resistance should be of little concern, since even if this type of resistance appears, it is impossible for cells to manifest fully malignant phenotypes, particularly in regards to invasion and metastasis potential.

\section{Acknowledgements}

We thank Dr Kevin Kaster of Geron Corp. for generously providing the plasmid pGRN145 that contained the fulllength hTERT complementary DNA (cDNA). This research was supported by grants from the National Nature Science Foundation of China (30470797, 30871150). 


\section{References}

1. Harley CB, Futcher AB and Greider CW: Telomeres shorten during ageing of human fibroblasts. Nature 345: 458-460, 1990.

2. Shay JW and Wright WE: Telomeres are double-strand DNA breaks hidden from DNA damage responses. Mol Cell 14: 420-421, 2004

3. Greider CW and Blackburn EH: Identification of a specific telomere terminal transferase activity in Tetrahymena extracts. Cell 43: 405-413, 1985.

4. Collins K: The biogenesis and regulation of telomerase holoenzymes. Nat Rev Mol Cell Biol 7: 484-494, 2006.

5. Kim NW, Piatyszek MA, Prowse KR, Harley CB, West MD, Ho PL, Coviello GM, Wright WE, Weinrich SL and Shay JW: Specific association of human telomerase activity with immortal cells and cancer. Science 266: 2011-2015, 1994.

6. Stewart SA and Weinberg RA: Telomerase and human tumorigenesis. Semin Cancer Biol 10: 399-406, 2000.

7. Yang SM, Fang DC, Luo YH, Lu R, Yang JL and Liu WW: Determination of telomerase activity and its subunits in different gastric lesions. Chin J Cancer 20: 23-27, 2001.

8. Yang SM, Fang DC, Luo YH, Lu R, Battle PD and Liu WW: Alterations of telomerase activity and terminal restriction fragment in gastric cancer and its premalignant lesions. J Gastroenterol Hepatol 16: 876-882, 2001.

9. Chen L, Cheng T, Cai YG, Tang XD, Li JJ, Fang DC, Luo YH, Yu ST and Yang SM: Expression of hTERT and its associated genes in the tumorigenesis of gastric mucosa and its significance. Chin J Gastroenterol Hepatol 15: 443-447, 2006.

10. Bryan TM, Englezou A, Dalla-Pozza L, Dunham MA and Reddel RR: Evidence for an alternative mechanism for maintaining telomere length in human tumors and tumorderived cell lines. Nat Med 3: 1271-1274, 1997.

11. Bryan TM, Englezou A, Gupta J, Bacchetti S and Reddel RR: Telomere elongation in immortal human cells without detectable telomerase activity. EMBO J 14: 4240-4248, 1995.

12. Muntoni A and Reddel RR: The first molecular details of ALT in human tumor cells. Hum Mol Genet 14: R191-R196, 2005.

13. Tsai HJ, Huang WH, Li TK, Tsai YL, Wu KJ, Tseng SF and Teng SC: Involvement of topoisomerase III in telomeretelomere recombination. J Biol Chem 281: 13717-13723, 2006.

14. Henson JD, Hannay JA, McCarthy SW, Royds JA, Yeager TR, Robinson RA, Wharton SB, Jellinek DA, Arbuckle SM, Yoo J Robinson BG, Learoyd DL, Stalley PD, Bonar SF, Yu D, Pollock RE and Reddel RR: A robust assay for alternative lengthening of telomeres in tumors shows the significance of alternative lengthening of telomeres in sarcomas and astrocytomas. Clin Cancer Res 11: 217-225, 2005.

15. Chang S, Khoo CM, Naylor ML, Maser RS and DePinho RA: Telomere-based crisis: functional differences between telomerase activation and ALT in tumor progression. Gene Dev 17: 88-100, 2003.

16. Canela A, Martin-Caballero J, Flores JM and Blasco MA: Constitutive expression of tert in thymocytes leads to increased incidence and dissemination of T-cell lymphoma in Lck-Tert mice. Mol Cell Biol 24: 4275-4293, 2004.
17. Stewart SA, Hahn WC, O'Connor BF, Banner EN, Lundberg AS, Modha P, Mizuno H, Brooks MW, Fleming M, Zimonjic DB, Popescu NC and Weinberg RA: Telomerase contributes to tumorigenesis by a telomere length-independent mechanism. Proc Natl Acad Sci USA 99: 12606-12611, 2002.

18. Sun BC, Huang Q, Liu SX, Chen MZ, Hawks CL, Wang LS, Zhang $\mathrm{CH}$ and Hornsby PJ: Progressive loss of malignant behavior in telomerase-negative tumorigenic adrenocortical cells and restoration of tumorigenicity by human telomerase reverse transcriptase. Cancer Res 64: 6144-6151, 2004.

19. Chen L, Tang XD, Yu ST, Ai ZH, Fang DC, Cai YG, Luo YH, Liang GP and Yang SM: Induction of anti-tumor immunity by dendritic cells transduced with hTERT recombinant adenovirus in mice. J Pathol 217: 685-692, 2009.

20. Cawthon RM: Telomere measurement by quantitative PCR. Nucleic Acids Res 30: e47, 2002.

21. Yang SM, Fang DC, Yang JL, Chen L, Luo YH and Liang GP: Antisense human telomerase reverse transcriptase could partially reverse malignant phenotypes of gastric carcinoma cell line in vitro. Eur J Cancer Prev 17: 209-217, 2008.

22. Yang SM, Fang DC, Yang JL, Liang GP, Lu R, Luo YH and Liu WW: Effect of antisense human telomerase RNA on malignant phenotypes of gastric carcinoma. J Gastroenterol Hepatol 17: 1144-1152, 2002.

23. Zhang G, Long M, Wu ZZ and Yu WQ: Mechanical properties of hepatocellular carcinoma cells. World J Gastroenterol 8: 243-246, 2002.

24. Wu ZZ, Zhang G, Long M, Wang HB, Song GB and Cai SX: Comparison of the viscoelastic properties of normal hepatocytes and hepatocellular carcinoma cells under cytoskeletal perturbation. Biorheology 37: 279-290, 2000.

25. Smith LL, Coller HA and Roberts JM: Telomerase modulates expression of growth-controlling genes and enhances cell proliferation. Nat Cell Biol 5: 474-479, 2003.

26. Geserick C, Tejera A, Gonzalez-Suarez E, Klatt P and Blasco MA: Expression of mTert in primary murine cells links the growthpromoting effects of telomerase to transforming growth factorbeta signaling. Oncogene 25: 4310-4319, 2006.

27. Bollmann FM: The many faces of telomerase: emerging extratelomeric effects. Bioessays 30: 728-732, 2008.

28. Bagheri S, Nosrati M, Li S, Fong S, Torabian S, Rangel J, Moore DH, Federman S, Laposa RR, Baehner FL, Sagebiel RW, Cleaver JE, Haqq C, Debs RJ, Blackburn EH and KashaniSabet M: Genes and pathways downstream of telomerase in melanoma metastasis. Proc Natl Acad Sci USA 103: 11306-11311, 2006.

29. Yang JM, Peng ZH, Si SH, Liu WW, Luo YH and Ye ZY: KAI1 gene suppresses invasion and metastasis of hepatocellular carcinoma MHCC97-H cells in vitro and in animal models. Liver Int 1: 132-139, 2008. 